
\title{
28 Research Square \\ Lipidomics of Facial Sebum in the Comparison Between Acne and Non-Acne Adolescents with Dark Skin
}

\section{Emeka Okoro}

Dermatology Unit, Federal Medical Centre, Keffı Nasarawa State

\section{Adebomi Adenle}

Dermatology Unit, Federal Medical Centre, Keffi Nasarawa State

Matteo Ludovici

San Gallicano Dermatological Institute- IRCCS

Mauro Truglio

San Gallicano Dermatological Institute- IRCCS

\section{Federico Marini}

'La Sapienza' University

Emanuela Camera ( $\nabla$ emanuela.camera@ifo.gov.it )

San Gallicano Dermatological Institute- IRCCS

\section{Research Article}

Keywords: Fatty acidomics, branched chain fatty acids, cholesterol, squalene, triglycerides, wax esters

Posted Date: May 20th, 2021

DOI: https://doi.org/10.21203/rs.3.rs-535091/v1

License: (c) (i) This work is licensed under a Creative Commons Attribution 4.0 International License. Read Full License

Version of Record: A version of this preprint was published at Scientific Reports on August 16th, 2021. See the published version at https://doi.org/10.1038/s41598-021-96043-x. 


\section{Abstract}

Lipidomics is advantageous in the study of sebum perturbations occurring in acne. An extended evaluation of the sebum lipid profiles in acne-prone sebaceous areas is lacking in dark skin. Yet, there is a void space in understanding how the building blocks of sebum lipids, i.e. individual fatty acids (FAs), are intertwined with acne-prone skin. We aimed to determine the sebum lipidome in facial areas of adolescents with and without acne in Nigeria. A cross-sectional analytical study was conducted in 60 adolescents/young adults divided in 30 acne patients (15F,15M) and 30 age and sex-matched controls. Sebum samples obtained from foreheads and cheeks were analysed separately by gas chromatographymass spectrometry (GCMS) and thin layer chromatography (HPTLC). Distributions of sebum components were investigated with multivariate ANOVA-simultaneous component analysis (ASCA). Sebum incretion in acne was paralleled by significantly higher abundance of triglycerides, wax esters, and squalene together with monounsaturated FAs (MUFAs), and straight chain saturated FAs (SFAs), especially those with oddcarbon chain, i.e. C13:0, C15:0, and C17:0. Profiling weight/weight percentage individual components revealed that in acne the free FAs (FFAs) array was shifted towards higher relative abundance of the SFAs C15:0, C16:0, and C17:0 and lower percentage of the anteiso-branched FFAs with 12, 14, 16, and 18 carbons. In acne patients, MUFAs and PUFAs were quantitatively increased and decreased on foreheads and cheeks, respectively. Relative abundance of fatty alcohols was decreased in acne independent on the site. The results indicated that acne associates with site-specific derangement of the pathways regulating the balance among odd straight-chain and branched-chain SFAs, MUFAs, including sapienate (C16:1n10), PUFAs, and squalene.

\section{Introduction}

Acne vulgaris is a chronic disorder of the pilosebaceous unit characterized by hyperseborrhea, dyseborrhea, skin dysbiosis, hyperkeratinisation, and inflammation, which altogether concur to the development of acne lesions ${ }^{1}$. The prevalence among adolescents varies between 30 and $90 \%$ in various population groups and geographical areas in the world ${ }^{2-5}$. Acne is a common reason for dermatological consult among adolescents with dark skin ${ }^{6}$. Increased sebum secretion is one of the multiple factors involved in the pathogenesis of acne. Positive correlations exist between sebum secretion rates and lesion counts ${ }^{7-11}$. Derangement in the lipid composition is an important contributor to the pathogenicity of sebum. Deficit of linoleic acid ${ }^{12}$, increase of overall percentage of free fatty acids (FFAs) and promotion of squalene peroxidation ${ }^{13-15}$, are accounted among the triggers for acne. The unbalanced triglycerides (TGs) to wax esters (WEs) ratio ${ }^{16,17}$ and higher levels of monounsaturated fatty acids (MUFAs) ${ }^{18}$ characterize acne sebum. Pappas et al. ${ }^{19}$ reported that squalene levels were higher in acne patients while our group reported higher levels of diglycerides (DGs) in sebum of adolescents with acne ${ }^{20}$. Density of sebaceous glands (SGs) and hormone receptors, together with gender, likely contribute to the different patterns of acne. Sex-related differences in the skin surface lipids composition have been observed in some cases and not observed in other ones ${ }^{21-23}$. Skin lipid composition is broadly shared among White, Asian, and African ethnicities $22,24,25$. However, there is a gap in the 
knowledge on biological characteristics of sebum production in ethnic patients. Distribution of sebum on the face is uneven with higher levels in the T-zone (forehead-nose-chin) compared to the U-zone (cheekbones-cheeks-jaw). In keeping with previous evidence ${ }^{11,26}$, we have reported a correlation of sebum levels with acne lesions in the U-zone not existing in the T-zone among adolescents with acne in Nigeria ${ }^{10}$. These observations could be related to the variation in sebum composition in the facial zones. The complexity of sebum and the multiplicity of the sebogenic pathways potentially involved in acne call for an extended and simultaneous detection of sebum components. There are no studies that compared sebum components in different facial areas in skin of colour. Our aim was to determine the profiles of abundance of sebum lipids in acne patients and unaffected controls of both genders in Nigeria.

\section{Results}

\section{Demographics and acne grading}

Demographics and distribution of the counted lesions of acne patients are detailed in the Supplementary Table S1. The mean age (years) of the acne patients and of controls was $17.2 \pm 1.76$, and 17.5 \pm 1.76 , respectively. Among the affected group, $15(50 \%)$ had mild acne while $10(33.3 \%)$ and $5(16.7 \%)$ had moderate and severe acne respectively.

\section{Determination of elemental components of facial sebum}

Forty-one FFAs, and 7 FOHs were quantified by GCMS (Supplementary Table S2). FFAs consisted of saturated and unsaturated species. Among saturated FFAs, several branched FFAs with one methyl branching at the iso (ibrFA) and anteiso (abrFA) positions were chromatographically resolved from the corresponding straight chain isomer with the same length. The latter ones were categorized as even (eFA) and odd (oFA) FFAs according to their even or odd number of $\mathrm{C}$-atoms. The ibrFAs, and abrFAs subgroups included 7 members each, with $\mathrm{C}$-atoms ranging from 12 to 18 . The subclasses of eFAs, oFAs, MUFAs, and PUFAs included 8, 7, 10, and 2 members, respectively. The eFAs and oFAs together were SFAs with chain length ranging from 12 to 26 carbon atoms. MUFAs had mainly an even C-number, with the exception of $\mathrm{C} 15: 1$, and $\mathrm{C17}: 1$. The latter one was found in both straight and branched isomers, which were assigned tentatively according to GCMS criteria. The C16:1 MUFA was characterized as sapienate $(\mathrm{C} 16: 1 \mathrm{n}-10)$ based on the same elution time and MS spectrum of the authentic reference compound and the resolution from palmitoleate (C16:1n-7), which eluted later. Palmitoleate was excluded from the quantitation due to its negligible amounts in sebum. The C18:1 MUFA appeared as a single peak. Likely, C18:1 isomers were not separated under the used GCMS conditions. FOHs with a chain length between 14 and 26 carbon atoms, were either confirmed with authentic compounds or tentatively assigned based on their MS spectrum and elution time (Supplementary Table S2). To visualize the within-class distributions of FFAs and FOHs, their amounts on foreheads and cheeks were averaged and plotted in radar diagrams (Supplementary Figure S3). Polarization of FFAs and FOHs distribution was apparent in both NA and A groups. The top 3 members, ordered according to their abundance, were, in each subclass, as follows: 13Me-C15:0 > 14Me-C16:0 > 12Me-C14:0 for the ibrFAs; 12Me-C15:0 > 15Me-C18:0 > 13Me- 
C16:0 for the abrFAs; C16:0 > C18:0 >> C14:0 for eFAs; C15:0 > C17:0 > C21:0 for the oFAs; C18:1 > C16:1 $>$ C17:1 for the MUFAs, and FOH18:0 >> FOH16:0 > FOHC22:0 for the FOHs. The PUFA C18:2 was more abundant than C20:2.

Separation of sebum lipids by HPTLC developed bands due to six major components, cholesterol, FFAs, TGs, WEs, CEs, and squalene (Supplementary Figure S1), according to their elution order. Heat maps of the average amounts of sebum lipids in foreheads and cheeks in NA and A groups are reported in the Figure $1 \mathrm{~A}$ and $1 \mathrm{~B}$, respectively. It is apparent that the amounts of most sebum lipids were significantly elevated in the A group. Inspection of the abundance profiles revealed that specific FFAs and FOHs members were affected more than others in the two sites. The supplementary Table S3 reports the amounts of sebum lipids (average $\mu \mathrm{g} \pm \mathrm{SD}$ ) represented in the heat maps.

\section{Sebum excretion rates and correlation of sebum lipids with acne lesions}

Sebum weights were obtained by summing the amounts $(\mu \mathrm{g})$ of individual lipids quantified by GCMS and HPTLC and were used to obtain the sebum excretion rates (SER), which expresses the $\mu \mathrm{g}$ secreted on surface unit per minute $(\mu \mathrm{g} / \mathrm{cm} 2 / \mathrm{min})$. SER values were significantly higher in acne patients (supplementary Figure S2) at both face sites, as shown by the p-values in the table included. SER values determined from cheeks were higher than those obtained from foreheads in the affected group only $(p<0,01)$. The associations of average composition of sebum from foreheads and cheeks with lesion counts was investigated by Spearman's correlation. The amounts of squalene were positively correlated with the number of comedones $(R=0.47, p<0,05$, data not shown).

\section{ASCA analysis on the relative concentrations of individual lipids.}

ASCA analysis, which is described in Appendix 1, was performed on the data set after normalization of the absolute individual amounts by the total sebum quantity (weight/weight percentage). The results of SCA analysis for the effect of the acne condition on the relative amounts on cheeks and foreheads are reported in Figure 2. The percentage of two aibrFFAs, i.e. 9Me-C12:0, and 11Me-C14:0, was lower on the forehead; whereas the ibrFFAs 10Me-C12:0, and 16Me-C18:0 had lower percentage on foreheads and cheeks, respectively. SFAs, and several MUFAs showed significantly higher percentage in the A group limited to the forehead. Percentages of short and long chain SFAs, PUFAs, and cholesterol, were decreased in A at the cheeks. Several FOHs were present at lower percentage in A, on both sites.

Percentages of squalene and cholesterol were, respectively, higher and lower in the A group regardless the face site. Among the A subjects, outliers accounted for the $20 \%$ and $27 \%$ of the entire A group when scores were examined in the foreheads and the cheek lipid profiles, respectively. Taken together, the comedone count $(16,6 \pm 4,2)$ in the outlier group was significantly lower $(p<0,05)$ than the rest of the $A$ subjects $(24,4 \pm 10,4)$.

\section{Discussion}


This study is the first to analyse sebum composition among adolescents of African descent with and without acne using combined GCMS and HPTLC analyses and multivariate statistics. The size of the control and affected groups was consistent with that of studies on acne. The skin surface lipids from both foreheads and cheeks were dominated by sebaceous lipids, as demonstrated by the detection of components unique to sebum, i.e. squalene and WEs ${ }^{17,27}$. The calculated SER were consistent with our previous data based on sebum amounts determined gravimetrically on the sampling tapes ${ }^{20}$. The amounts of sebum components were unevenly distributed on the face of the A group, with cheeks bearing more sebum than foreheads. This observation is in contrast with the casual sebum levels determined with the SebumeterTM on the same sites and ethnic group with similar age and gender distribution ${ }^{10}$. The lack of studies comparing instrumental assessments with quantitative determination of sebum components limits conclusions ${ }^{28}$. The overall results demonstrated that the facial sebum secretion was higher in acne patients compared to age and gender matched controls, at both face sites, in keeping with established pathogenic factors of acne ${ }^{10}$. The higher amounts of sebum in acne were mirrored by significantly higher levels of individual lipids, i.e. squalene, cholesterol, and vitamin E, or lipid families, such as FFAs, FOHs, TGs, and WEs. Squalene levels correlated positively with the counts of noninflammatory lesions, in agreement with the occurrence of squalene peroxide and the depletion of vitamin $E$ in acne sebum ${ }^{17,29,30}$ and the patterns of acne distribution ${ }^{11}$. Among FFAs, most straight and branched chain FFAs showed significantly higher levels in acne sebum. Previous studies have focused on the bound FA originated from sebum lipids separated by HPTLC 19, 22, 31, 32. FFAs have been evaluated in different ethnicities, including the African ones, by LCMS ${ }^{25}, 33$. The LC methods commonly used do not resolve the separation of isobaric FFAs limiting the comparability of branched FFAs between acne patients and their controls. Pappas et al. reported a lower amount of FFAs in sebum of acne patients not specific to straight or branched chain FFA ${ }^{19}, 31,32$. We observed that several straight and branched chain FFAs (mostly with odd carbon-number, i.e. C13, C15, and C17) were among the upregulated species. Noteworthy, the abrFFAs were more affected than ibrFFAs. These differences were suggestive of abnormal synthesis of the odd-chain FFAs in acne. Opposite to other biofluids, branched chain FA are relatively abundant in sebum ${ }^{34}$. Multiple sources contribute to their level, such as de novo synthesis by the $S G$, metabolism of skin microbes, and diet ${ }^{35,36}$.

Canonical FA synthesis is initiated from acetyl-CoA. Propionic acid, produced either by microbial metabolism or amino acid catabolism ${ }^{37}$ ), and valeric acid, are the precursors of odd numbered straight chained C13:0, C15:0, C17:0, which are among the most upregulated FFAs in acne. Branched FAs are synthesized from branched starters like short chain organic acids, and aminoacids. In particular, isobutyrate and isovalerate acid are precursors of ibrFAs with even and odd C-number, respectively. Leucine and isoleucine are the precursors of ibrFFAs and aibrFFA, respectively, mostly with 15 and $17 \mathrm{C}$ atoms. Valine is the starter of the even-numbered ibrFAs ${ }^{38}$. Further studies are needed to understand the complex interconnections sebum FFAs establish with short chain organic acids, and amino acid metabolism. 
The sebaceous-type desaturation pathways of FFAs appeared to be relevant in acne. Induction of MUFAs with sebum-type chain length of 14-17 C-atoms was very significant at both sites. MUFAs with epidermal-type chain length, i.e. 18-24 C-atoms, were induced at a lesser extent. Incretion was more pronounced at cheeks. The sebaceous gland expresses both fatty acid desaturase 2 (FADS2) and stearoyl-CoA desaturase 1 (SCD-1). FADS2 is a $\Delta 6$ desaturase that converts palmitate into sapienate (C16:1n-10) following the insertion of a C-C double bond at the position 6. SCD-1 is a $\Delta 9$ desaturase showing substrate preference towards stearate, which is converted into oleate (C18:1n-9) however it can also yield palmitoleate (C16:1n-7) from palmitate. In our approach, the prevalent sapienate isomer could be clearly discriminated from palmitoleate, which was a negligible MUFA in sebum. In contrast, assignments of C18:1 isomers was ambiguous. The current knowledge is rather limited to exclude that stearate undertakes both desaturation roots in the sebaceous gland. Recent evidence have demonstrated that FADS2 inserts a $\triangle 6$ double bond in the branched and straight C17:0 ${ }^{39}$. GCMS profiles showed peaks tentatively assigned as branched and straight C17:1 MUFAs. Enhanced MUFAs/SFAs ratio for C16, C17, and $\mathrm{C} 18$ chains in acne were suggestive of promotion of multiple desaturation pathways. Multivariate analysis by means of ASCA revealed that straight FFAs and branched FFAs with even carbon-number were increased at a lesser extent than those with odd-chain length. One possibility is that the availability of precursors of even-chain branched FFAs can be limited compared to that of precursors of odd-chain branched FFAs. The lower weight/weight percentage of ibrFAs and aibrFAs with even-chain in acne sebum might suggest that the de novo synthesis of these FFAs is left behind in competitive biosynthetic pathways leading to straight chain oFAs and eFAs. The profiles of relative abundance also revealed a shift towards higher MUFAs in acne, especially on foreheads. This evidence reinforces the involvement of the FADS2 pathway in the promotion of the sebum synthesis and occurrence of acne. Enhanced FADS2 activity leading to higher $\mathrm{C} 16: 1 / \mathrm{C} 16: 0$ ratio has been implicated in the acnegenic effects of high glycaemic loads diets ${ }^{18,40}$. The lower levels of FA C18:2 found in acne sebum agrees with the deficit of linoleic acid reported in previous studies ${ }^{12,41}$. Profiles of abundance of $\mathrm{C} 20: 2$ paralleled those observed for C18:2 suggesting that these two PUFAs are related metabolites. The higher levels of squalene and lower amounts of cholesterol in A suggests a possible further inhibition of post-squalene cholesterol biosynthesis in the SG. For the first time, FOHs were examined for their impact on sebum composition in healthy and acne sebum. FOHs bind together with FAs to form WEs. Although pathways of FOHs and FFAs biosynthesis are intersected, the knowledge on the steps preceding the FOHs biosynthesis in the SG is rather limited ${ }^{39}$. The overall data are in favour of uneven distribution of sebum components on the face. Overall, the differences in the levels of sebum lipid components between foreheads and cheeks were more pronounced in acne patients. The ASCA approach identified outliers in both NA and A groups, highlighting the need to stratify cases and recognize patterns of acne-like sebum profiles in absence of clinical manifestations. The differences found between foreheads and cheeks may be related to distribution, size and activity of the SGs 42,43 and may partly explain the patterns of inflammatory dermatoses on the T- and U-zones ${ }^{10}$. In particular, the data suggested a different impact of the disorder on multiple pathways leading to the FFAs isomers. 


\section{Methods}

\section{Ethical consideration}

The study was performed in agreement with the Declaration of Helsinki principles. Ethical approval was obtained from the Research Ethics Committee of the Federal Medical Center, Keffi Nasarawa State before commencing the study. An informed consent was obtained from the participants or their guardians (if the participant was underage) before enrolment in the study.

\section{Study design}

A cross-sectional analytical study was conducted in adolescents and young adults that volunteered to participate in the study. The volunteers were selected by a non-randomized method following advertisement of the study in the university and senior secondary schools. Patients receiving treatment for acne vulgaris, those that suspended topical or systemic treatments earlier than two weeks (four weeks for patients that used retinoids) and patients with other skin or systemic diseases that could interfere with sebum analysis were excluded from the study. Enrolment was concluded when sixty participants (30 acne patients and 30 controls) that met the requirements were recruited. Equal number of females $(F)$ and males (M) were recruited in both groups with age ranging between 15 and 25 years. The face of acne patients was examined. The types of acne lesions on the face, i.e. non inflammatory (comedones), and inflammatory ones (papules, pustules and nodules), were counted and documented. The severity of acne was rated as mild (1, predominance of comedones and a few inflammatory lesions), moderate (2, some comedones, and mainly papules, and pustules), and severe (3, predominance of papules, pustules and nodules).

\section{Materials, chemicals, and reagents}

Sebutapes $^{\mathrm{TM}}$ and storage cards were purchased from CuDerm Corporation (Dallas, TX, US). Authentic lipid standards were purchased from several manufacturers as detailed in Supplementary Table S2. Other details of the lipid standards are provided in the table. The chemicals to prepare the solution of BSTFA in pyridine containing $1 \%$ trimethylchlorosilane (TCMS) were from Merck (Darmstadt, Germany).

\section{Sebum sampling}

Sebum was collected with the Sebutapes ${ }^{\mathrm{TM}}$ adhesive patches. Two patches were placed onto the centre of the forehead and two patches were applied onto the most prominent area of the cheeks, one on each side, to absorb sebum for 30 minutes. The patches were removed and placed on storage cards, which were labelled appropriately as non-acne controls (NA1-NA30) and acne cases (A1-A30). The cards were stored refrigerated until processing.

\section{Sample preparation and sebum analysis}


Sebum analysis was carried out separately on specimens obtained from the foreheads and the cheeks. To perform the extraction of the study samples and blank tapes, clean glass-tubes were pre-loaded with $200 \mu \mathrm{L}$ of the internal standard (ISTD) mixture (d6cholesterol $100 \mu \mathrm{M}$, d6squalene $100 \mu \mathrm{M}$, and d17C16:0 $50 \mu \mathrm{M}$ in ethanol) and stored at $-20^{\circ} \mathrm{C}$ until use. Sebum lipids were extracted from the tapes as previously reported ${ }^{20}$. Details are provided in Supplementary material (Appendix 1). The dry extract was dissolved in $500 \mu \mathrm{L}$ of acetone/methanol/isopropanol (40/40/20 v/v/v) mixture to be analyzed by gas chromatography coupled to mass spectrometry (GCMS) and high-performance thin layer chromatography (HPTLC) to determine the target lipid species as detailed in Appendix 1. For the quantitative analyses of individual lipids by GCMS we constructed calibration curves of the authentic standards listed in the Supplementary Table S2, representing FFAs, fatty alcohols (FOHs), squalene, cholesterol, and vitamin $E$ in sebum. The molecular ions and the fragments accounted for the quantitative GCMS assessments are summarized in the Supplementary Table S2. Amounts of putative known lipids, i.e. FOH C20:0, $\mathrm{FOH} \mathrm{C22:0,} \mathrm{and} \mathrm{FOH} \mathrm{C24:0,} \mathrm{were} \mathrm{referred} \mathrm{to} \mathrm{the} \mathrm{structurally} \mathrm{closest}$ compound available as an authentic standard. Total TGs, WEs, and cholesterol esters (CEs) were assessed quantitatively by HPTLC, against reference lipids loaded at different concentration on the same plate together with the sebum samples (Appendix 1). Representative HPTLC and GCMS chromatograms are reported in Supplementary Figure S1.

Data were analysed using the statistical and data analysis solutions XLSTAT 2020.1.2 (Addinsoft, New York, USA), and MatLab (version 8.6.0 release R2015b; The Mathworks, Natick, MA). Continuous variables were represented as median values with confidence intervals or mean \pm standard deviation (SD). MannWhitney and Kruskal-Wallis tests were used for comparison between two or more groups. Spearman's coefficient $(R)$ was used to measure the correlation between two quantitative variables. Differences and correlations were considered statistically significant with $p \leq 0.05$. ANOVA-simultaneous component analysis (ASCA) ${ }^{44}$, was used to determine the effect of the controlled factors in the experimental design on the multivariate chromatographic profiles, as described in the supplementary material (Appendix 1).

\section{Declarations}

\section{Acknowledgements}

This study was funded by research grant from Fondation Rene Touraine and supported by resources of the Laboratory for Cutaneous Physiopathology, San Gallicano Dermatological Institute, Rome, Italy

\section{Author contribution}

EO, AA, and EC conceived the study; EO and ML applied the methodology and conducted the experiments; EO, EC, and MT curated the data; MT, FM validated the data and performed formal analysis; EO, FM, and EC wrote the manuscript. All Authors reviewed the manuscript.

\section{References}


1. Layton AM. in Rook's text book of dermatology (ed Burns T, Breathnach S, Cox N, Griffiths C) 20012070 (Wiley-Blackwell, 2010).

2. Ogunbiyi, A. O., Omigbodun, Y. \& Owoaje, E. Prevalence of skin disorders in school children in southwest Nigeria. Int. J. Adolesc. Med. Health 21, 235-241 (2009).

3. Yahya, H. Acne vulgaris in Nigerian adolescents-prevalence, severity, beliefs, perceptions, and practices. Int. J. Dermatol. 48, 498-505 (2009).

4. Ghodsi, S. Z., Orawa, H. \& Zouboulis, C. C. Prevalence, severity, and severity risk factors of acne in high school pupils: a community-based study. J. Invest. Dermatol. 129, 2136-2141 (2009).

5. Okoro, O., Ogunbiyi, A. \& George, A. Prevalence and pattern of acne vulgaris among adolescents in Ibadan, south-west Nigeria. J Egypt. Women Dermatol. Soc. 13, 7-12 (2016).

6. Zouboulis, C., Okoro, O. \& Kubba, R. in Pigmented Ethnic Skin and Imported Dermatoses: A Text-Atlas (eds Orfanos, C., Zouboulis, C. \& Assaf, C.) 203-218 (Springer Nature, Switzerland:, 2018).

7. Harris HH, Downing DT, Stewart ME \& Strauss JS. Sustainable rates of sebum secretion in acne patients and matched normal control subjects. J. Am. Acad. Dermatol. 8, 200-3 (1983).

8. Youn, S. W., Park, E. S., Lee, D. H., Huh, C. H. \& Park, K. C. Does facial sebum excretion really affect the development of acne? Br. J. Dermatol. 153, 919-924 (2005).

9. Kim, M. K. et al. Comparison of sebum secretion, skin type, $\mathrm{pH}$ in humans with and without acne. Arch. Dermatol. Res. 298, 113-119 (2006).

10. Okoro, E. O., Bulus, N. G. \& Zouboulis, C. C. Study of Facial Sebum Levels and Follicular Red Fluorescence in Patients with Acne Vulgaris in Nigeria. Dermatology 232, 156-161 (2016).

11. Choi, C. W., Choi, J. W., Park, K. C. \& Youn, S. W. Facial sebum affects the development of acne, especially the distribution of inflammatory acne. J. Eur. Acad. Dermatol. Venereol. 27, 301-306 (2013).

12. Downing, D. T., Stewart, M. E., Wertz, P. W. \& Strauss, J. S. Essential fatty acids and acne. J. Am. Acad. Dermatol. 14, 221-225 (1986).

13. Kanaar, P. Follicular-keratogenic properties of fatty acids in the external ear canal of the rabbit. Dermatologica 142, 14-22 (1971).

14. Motoyoshi, K. Enhanced comedo formation in rabbit ear skin by squalene and oleic acid peroxides. Br. J. Dermatol. 109, 191-198 (1983).

15. Chiba K, Yoshizawa K, Makino I, Kawakami K \& Onoue M. Comedogenicity of squalene monohydroperoxide in the skin after topical application. J. Toxicol. Sci. 25, 77-83 (2000).

16. Stewart ME, Steele WA \& Downing DT. Changes in the relative amounts of endogenous and exogenous fatty acids in sebaceous lipids during early adolescence. J. Invest. Dermatol. 92, 371-378 (1989).

17. Picardo M, Ottaviani M, Camera E \& Mastrofrancesco A. Sebaceous gland lipids. DermatoEndocrinology 1, 68-71 (2009). 
18. Smith, R. N., Braue, A., Varigos, G. A. \& Mann, N. J. The effect of a low glycemic load diet on acne vulgaris and the fatty acid composition of skin surface triglycerides. J. Dermatol. Sci. 50, 41-52 (2008).

19. Pappas A, Johnsen S, Liu JC \& Eisinger M. Sebum analysis of individuals with and without acne. Dermato-Endocrinology 1, 157-61 (2009).

20. Camera, E. et al. Use of lipidomics to investigate sebum dysfunction in juvenile acne. J. Lipid Res. 57, 1051-1058 (2016).

21. Cotterill, J. A., Cunliffe, W. J., Williamson, B. \& Bulusu, L. Age and sex variation in skin surface lipid composition and sebum excretion rate. Br. J. Dermatol. 87, 333-340 (1972).

22. Shetage, S. S. et al. Effect of ethnicity, gender and age on the amount and composition of residual skin surface components derived from sebum, sweat and epidermal lipids. Skin Res. Technol. 20, 97107 (2014).

23. Ramasastry, P., Downing, D. T., Pochi, P. E. \& Strauss, J. S. Chemical composition of human skin surface lipids from birth to puberty. J. Invest. Dermatol. 54, 139-144 (1970).

24. Yamamoto, A., Serizawa, S., Ito, M. \& Sato, Y. Effect of aging on sebaceous gland activity and on the fatty acid composition of wax esters. J. Invest. Dermatol. 89, 507-512 (1987).

25. Shetage, S. S., Traynor, M. J., Brown, M. B. \& Chilcott, R. P. Sebomic identification of sex- and ethnicity-specific variations in residual skin surface components (RSSC) for bio-monitoring or forensic applications. Lipids Health. Dis. 17, 194-018-0844-z (2018).

26. Choi, C. W., Choi, J. W., Park, K. C. \& Youn, S. W. Ultraviolet-induced red fluorescence of patients with acne reflects regional casual sebum level and acne lesion distribution: qualitative and quantitative analyses of facial fluorescence. Br. J. Dermatol. 166, 59-66 (2012).

27. Smith, K. R. \& Thiboutot, D. M. Thematic review series: skin lipids. Sebaceous gland lipids: friend or foe? J. Lipid Res. 49, 271-281 (2008).

28. Voegeli, R., Gierschendorf, J., Summers, B. \& Rawlings, A. V. Facial skin mapping: from single point bio-instrumental evaluation to continuous visualization of skin hydration, barrier function, skin surface pH, and sebum in different ethnic skin types. Int. J. Cosmet. Sci. 41, 411-424 (2019).

29. Capitanio, B. et al. 'Smoker's acne': a new clinical entity? Br. J. Dermatol. 157, 1070-1071 (2007).

30. Capitanio B et al. Modulation of sebum oxidation and interleukin-1a levels associates with clinical improvement of mild comedonal acne. Journal of the European Academy of Dermatology and Venereology (Elsevier) (2014).

31. Pappas A, Fantasia J \& Chen T. Age and ethnic variations in sebaceous lipids. DermatoEndocrinology 5, 319-24 (2013).

32. Li, W. H. et al. In vitro modeling of unsaturated free fatty acid-mediated tissue impairments seen in acne lesions. Arch. Dermatol. Res. 309, 529-540 (2017).

33. Michael-Jubeli R, Tfayli A, Bleton J \& Baillet-Guffroy A. Chemometric approach for investigating the skin surface lipids (SSLs) composition: influence of geographical localization. European Journal of 
Dermatology 21, 63-71 (2011).

34. Taormina, V. M., Unger, A. L., Schiksnis, M. R., Torres-Gonzalez, M. \& Kraft, J. Branched-Chain Fatty Acids-An Underexplored Class of Dairy-Derived Fatty Acids. Nutrients 12, 10.3390/nu12092875 (2020).

35. Ran-Ressler, R. R. et al. Branched chain fatty acid content of United States retail cow's milk and implications for dietary intake. Lipids 46, 569-576 (2011).

36. Ran-Ressler, R. R., Bae, S., Lawrence, P., Wang, D. H. \& Brenna, J. T. Branched-chain fatty acid content of foods and estimated intake in the USA. Br. J. Nutr. 112, 565-572 (2014).

37. Crown, S. B., Marze, N. \& Antoniewicz, M. R. Catabolism of Branched Chain Amino Acids Contributes Significantly to Synthesis of Odd-Chain and Even-Chain Fatty Acids in 3T3-L1 Adipocytes. PLoS One 10, e0145850 (2015).

38. Wallace, M. et al. Enzyme promiscuity drives branched-chain fatty acid synthesis in adipose tissues. Nat. Chem. Biol. 14, 1021-1031 (2018).

39. Wang, Z. et al. Fatty acid desaturase 2 (FADS2) but not FADS1 desaturates branched chain and odd chain saturated fatty acids. Biochim. Biophys. Acta Mol. Cell. Biol. Lipids 1865, 158572 (2020).

40. Smith, R. N., Mann, N. J., Braue, A., Makelainen, H. \& Varigos, G. A. A low-glycemic-load diet improves symptoms in acne vulgaris patients: a randomized controlled trial. Am. J. Clin. Nutr. 86, 107-115 (2007).

41. Morello AM, Downing DT \& Strauss JS. Octadecadienoic acids in the skin surface lipids of acne patients and normal subjects. J. Invest. Dermatol. 66, 319-23 (1976).

42. Pierard, G. E., Pierard-Franchimont, C. \& Kligman, A. M. Kinetics of sebum excretion evaluated by the Sebutape-Chromameter technique. Skin Pharmacol. 6, 38-44 (1993).

43. Pierard, G. E. Follicule to follicule heterogeneity of sebum excretion. Dermatologica 173, 61-65 (1986).

44. Smilde, A. K. et al. ANOVA-simultaneous component analysis (ASCA): a new tool for analyzing designed metabolomics data. Bioinformatics 21, 3043-3048 (2005).

\section{Figures}




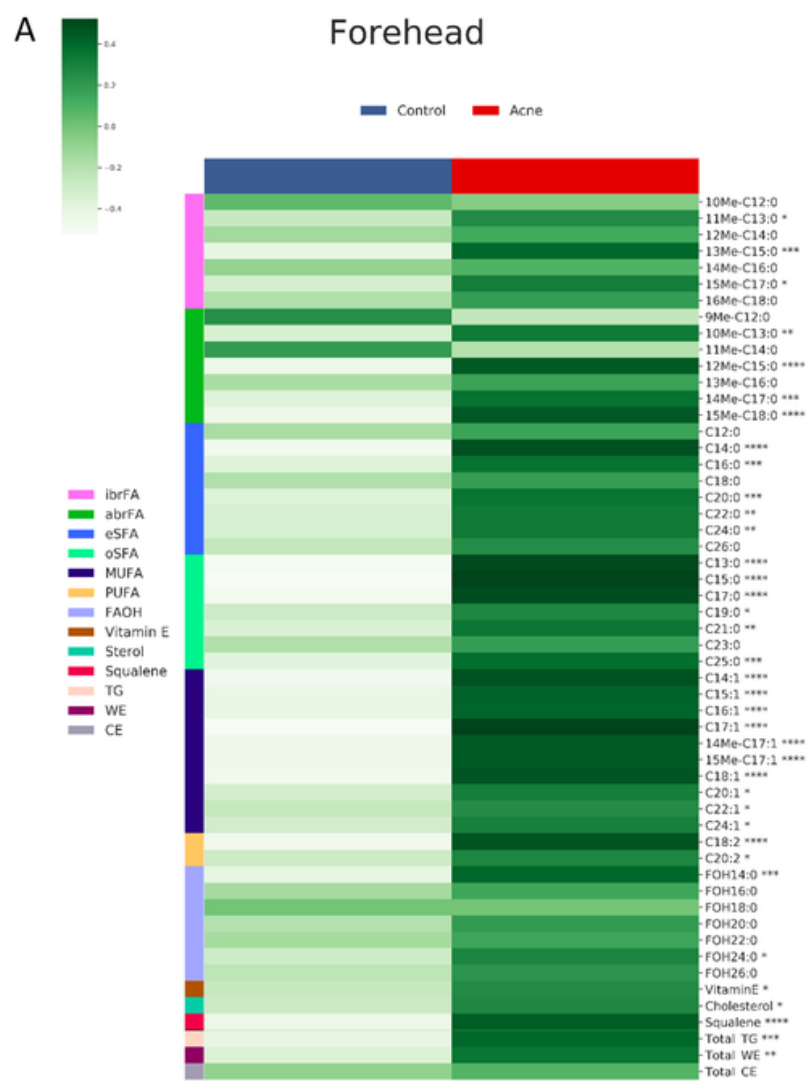

B

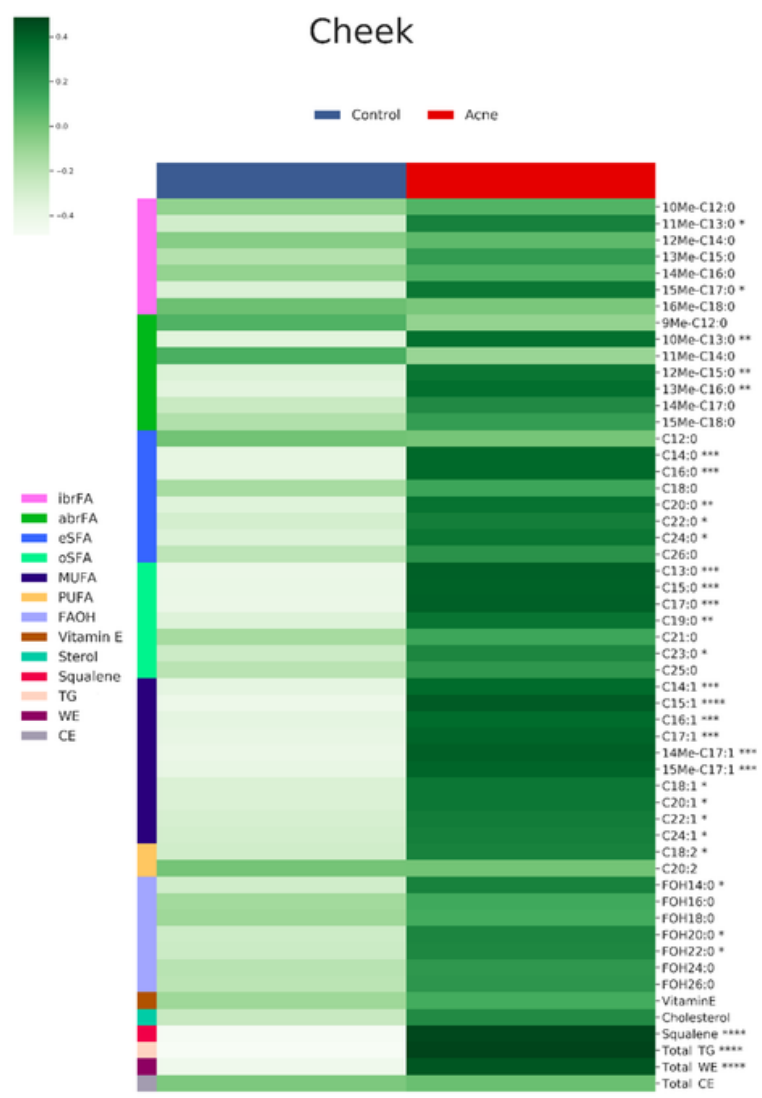

\section{Figure 1}

Heatmaps of amounts in $\mu \mathrm{g}$ of lipids quantified in sebum sampled with SebutapeTM patches on foreheads (A) and cheeks (B). Triglycerides (TGs), wax esters (WEs), and cholesterol esters (CEs) were quantified by HPTLC, whereas free fatty acids (FFAs), fatty alcohols (FOHs), squalene and cholesterol were quantified by GCMS. The total sebum amounts were obtained by summing the quantitative data obtained by HPTLC and GCMS. Significant differences were determined with Mann-Whitney test. ${ }^{*} p \leq 0.05,{ }^{* *} p<0.001,{ }^{* \star *} p<0.005, * \star * * p<0.0001$. 


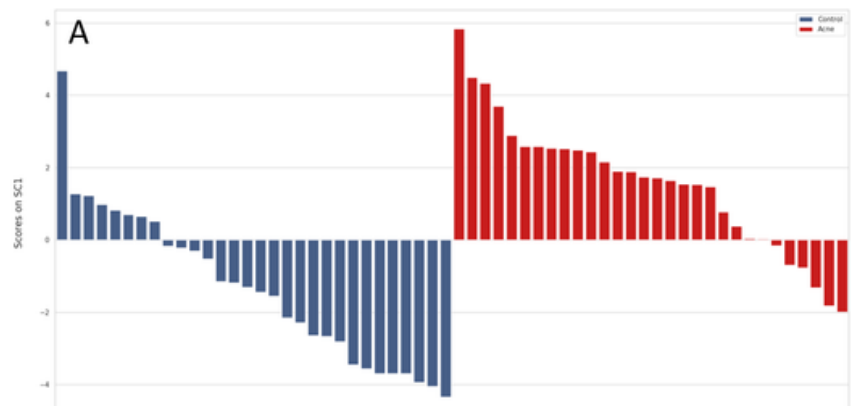

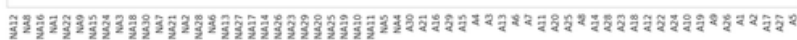
sonste

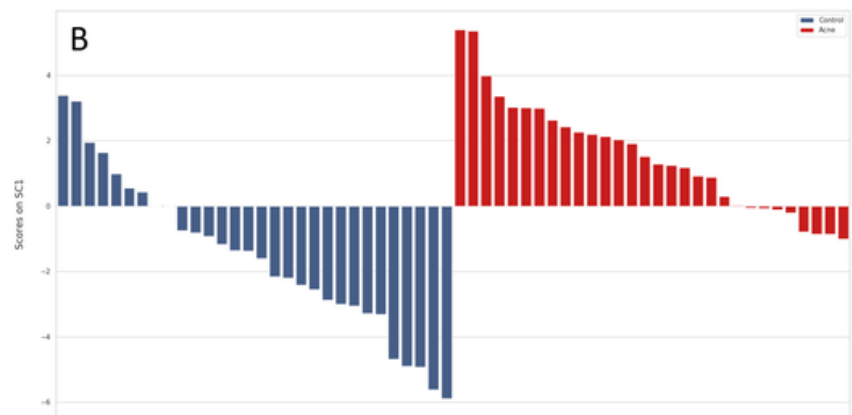

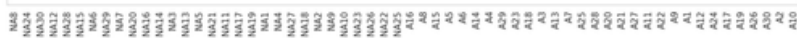
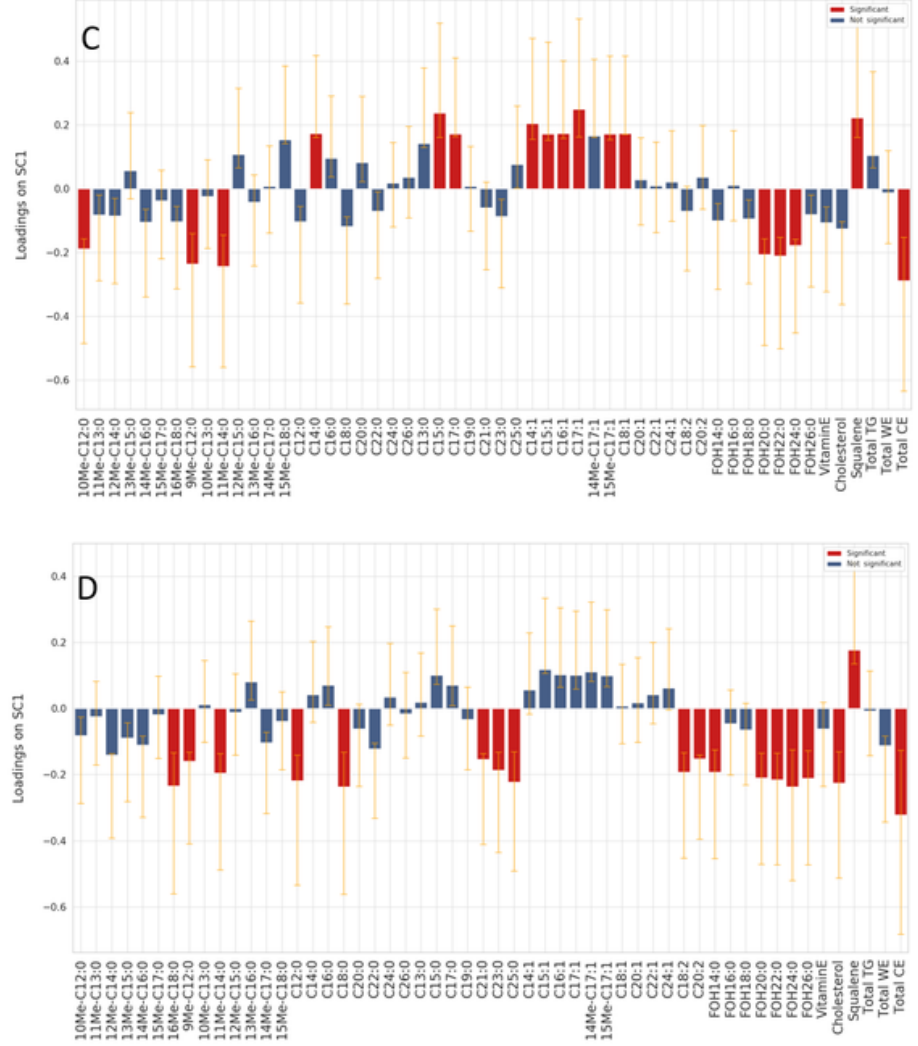

Figure 2

SCA analysis on the effect matrix (normalized quantities) for acne condition investigated in sebum from foreheads (A-C) and from cheeks (B-D). (A-B) Scores plots after projection of the residuals onto the space spanned by the only significant $\mathrm{PC}$; legend: blue = non-acne; red $=$ acne. $\mathrm{A}$ and $\mathrm{B}$ plots refer to the scores of foreheads and cheeks, respectively. (C-D) Variable loadings on SC1, together with their confidence interval (red and blue bars indicate significantly and not significantly contributing descriptors, respectively). C and D bar graphs refer to the loadings on the SC1 of individual lipids in foreheads and cheeks, respectively

\section{Supplementary Files}

This is a list of supplementary files associated with this preprint. Click to download.

- SupplementaryTableS1.docx

- SupplementaryTableS2.xlsx

- SupplementaryTableS3.docx

- SupplementaryFigureS1.pptx

- SupplementaryFigureS2.pptx

- SupplementaryFigureS3.pptx 
- AppendixS1.docx

Page 14/14 